\title{
MODULES DETERMINED BY THEIR ANNIHILATOR CLASSES
}

\author{
SIMION BREAZ AND JAN TRLIFAJ
}

\begin{abstract}
We present a classification of those finite length modules $X$ over a ring $A$ which are isomorphic to every module $Y$ of the same length such that $\operatorname{Ker}\left(\operatorname{Hom}_{A}(-, X)\right)=\operatorname{Ker}\left(\operatorname{Hom}_{A}(-, Y)\right)$, i.e. $X$ is determined by its length and the torsion pair cogenerated by $X$. We also prove the dual result using the torsion pair generated by $X$. For $A$ right hereditary, we prove an analogous classification using the cotorsion pair generated by $X$, but show that the dual result is not provable in $\mathrm{ZFC}$.
\end{abstract}

\section{INTRODUCTION}

Let $A$ be a ring. For a class of (right $A$-) modules $\mathcal{C}$, we consider the following annihilator classes

${ }^{\circ} \mathcal{C}=\left\{M \in \operatorname{Mod}-A \mid \operatorname{Hom}_{A}(M, \mathcal{C})=0\right\}, \quad \mathcal{C}^{\circ}=\left\{M \in \operatorname{Mod}-A \mid \operatorname{Hom}_{A}(\mathcal{C}, M)=0\right\}$, and

${ }^{\perp} \mathcal{C}=\left\{M \in \operatorname{Mod}-A \mid \operatorname{Ext}_{A}^{1}(M, \mathcal{C})=0\right\}, \quad \mathcal{C}^{\perp}=\left\{M \in \operatorname{Mod}-A \mid \operatorname{Ext}_{A}^{1}(\mathcal{C}, M)=0\right\}$.

The annihilator classes of the form ${ }^{\circ} \mathcal{C}$ for some $\mathcal{C} \subseteq$ Mod- $A$ are well-known to coincide with the torsion classes of modules, i.e., the classes closed under direct sums, extensions, and homomorphic images. Dually, $\mathcal{C}^{\circ}$ are the torsion-free classes, i.e., the classes closed under direct products, extensions, and submodules, [9, §VI.2].

The annihilator class ${ }^{\perp} \mathcal{C}\left(\mathcal{C}^{\perp}\right)$ is closed under direct summands, extensions, direct sums (direct products), and contain all projective (injective) modules, but it is not characterized by these closure properties in general (see Examples 1 and 2 below). This is the reason why it is hard to compute the annihilator classes of the form ${ }^{\perp} \mathcal{C}$ and $\mathcal{C}^{\perp}$ explicitly, and in some cases (e.g., for the class of all Whitehead groups ${ }^{\perp} \mathbb{Z}$ ), their structure depends on additional set-theoretic assumptions, cf. [3, Chap.XIII].

In this paper we address the more tractable problem of comparing rather than computing the annihilator classes, and of characterizing modules by their annihilator classes.

Recall that in particular cases, there are close relations among some of the annihilator classes. For example, if $\mathcal{C}$ consists of finitely presented modules of projective dimension $\leq 1$, then the classes $\mathcal{C}^{\perp}$ are exactly the tilting torsion classes of modules, $[6, \S 6.1]$. If moreover $A$ is an artin algebra then $\mathcal{C}^{\perp}$ are exactly the torsion classes closed under direct products, pure submodules, and containing all injective modules, cf. [2, 3.7]. In this case the Auslander-Reiten formula provides a precise

Date: July 9, 2009

2000 Mathematics Subject Classification. Primary: 16D70. Secondary: 16E30, 16S90, 03E35. Key words and phrases. Modules of finite length, torsion and cotorsion pairs.

$\mathrm{S}$. Breaz is supported by the grant PN2-ID489 (CNCSIS).

J. Trlifaj is supported by GAČR 201/09/0816 and MSM 0021620839. 
relation, namely $X^{\perp}={ }^{\circ}(\tau X)$ for each finitely presented module $X$ of projective dimension $\leq 1$ where $\tau$ denotes the Auslander-Reiten translation. Dually, if $Y$ is a finitely presented module of injective dimension $\leq 1$, then ${ }^{\perp} Y=\left(\tau^{-} Y\right)^{\circ}$, see $[1$, IV.2].

Surprisingly, the conditions ${ }^{\circ} X \subseteq{ }^{\circ} Y$ and $X^{\perp} \subseteq Y^{\perp}$ (and the dual ones) are closely related even for general modules. We show this by expressing these conditions in terms of existence of certain chains of submodules. Thus we prove equivalence of the two conditions for certain finite length modules (see Theorem 7 below).

Of course, in general we may have $X \not Y$ even if ${ }^{\circ} X={ }^{\circ} Y$ and $X^{\perp}=Y^{\perp}$ (just take $Y=X^{2}$ where $X$ is any non-zero module of finite length). Moreover, this is possible even if we impose the condition " $X$ and $Y$ have the same length": If $X$ is indecomposable such that $\operatorname{Ext}_{A}^{1}(X, X) \neq 0$, there exists $Y \neq X^{2}$ such that $X$ embeds in $Y$ and $Y / X \cong X$; it is not hard to see that ${ }^{\circ} X^{2}={ }^{\circ} Y$, and $X^{2 \perp}=Y^{\perp}$ if $A$ is hereditary (see also [8, Example 5.1]).

Developing further some of the ideas from [8] and [12], we characterize in Theorem 21 those modules $X$ of length $\lg (X)<\infty$ which are isomorphic to each finite length module $Y$ such that $\lg (X)=\lg (Y)$ and ${ }^{\circ} X={ }^{\circ} Y$. The corresponding version for $X^{\perp}=Y^{\perp}$ is proved in Theorem 24 assuming that $A$ is a right hereditary ring. The dual of Theorem 24 fails by Example 28. However, Theorem 21 can be dualized; this is proved in Theorem 27 .

\section{Comparing the annihilator Classes}

We start by two examples showing that unlike the classes of the form ${ }^{\circ} \mathrm{C}$ and $\mathcal{C}^{\circ}$, the annihilator classes ${ }^{\perp} \mathcal{C}$ and $\mathcal{C}^{\perp}$ are not characterized by their basic closure properties in general.

Example 1. This is an example of a class $\mathcal{D}$ of modules closed under direct summands, direct sums, extensions, and containing all projective modules, but such that $\mathcal{D} \neq{ }^{\perp} \mathcal{C}$ for any class of modules $\mathcal{C}$.

We consider the setting of (abelian) groups $(=\mathbb{Z}$-modules), and $\mathcal{D}$ will be the class of all $\aleph_{1}$-free groups (i.e., the groups $M$ such that each countable subgroup of $M$ is free). Clearly $\mathcal{D}$ contains all free groups, and it is closed under direct summands and extensions. The Baer-Specker theorem says that any direct product of copies of $\mathbb{Z}$ is $\aleph_{1}$-free (cf. [3, IV.2.8]). By [5, Lemma 1.2], if $C$ is a group such that $\operatorname{Ext}_{\mathbb{Z}}^{1}(P, C)=0$ for any direct product $P$ of copies of $\mathbb{Z}$, then $C$ is a cotorsion group, so ${ }^{\perp}\{C\}$ contains all torsion-free groups. In particular the group of all rational numbers $\mathbb{Q} \in{ }^{\perp}\{C\}$, but $\mathbb{Q}$ is not $\aleph_{1}$-free. So there is no class of groups $\mathcal{C}$ such that $\mathcal{D}={ }^{\perp} \mathcal{C}$.

Example 2. Now we give an example of a class $\mathcal{D}$ of modules closed under direct summands, direct products, extensions, and containing all injective modules, but such that $\mathcal{D} \neq \mathcal{C}^{\perp}$ for any class of modules $\mathcal{C}$. In this example, we will assume that there are no $\omega$-measurable cardinals (this holds under the Axiom of Constructibility $\mathrm{V}=\mathrm{L}$, for example, see [3, VI.3.14]).

We will work in the setting of (right $A$-) modules where $A$ is a simple non-artinian von Neumann regular ring such that $A$ has countable dimension over its center $K$. (Note that $K$ is a field by [7, Corollary 1.15]). For a concrete example of such ring, we can take $A=\lim _{\longrightarrow} M_{2^{n}}(K)$, the direct limit of the direct system of full matrix 
$K$-algebras where $K$ is a field and

$$
K \stackrel{f_{0}}{\hookrightarrow} M_{2}(K) \stackrel{f_{1}}{\hookrightarrow} \ldots \stackrel{f_{n-1}}{\hookrightarrow} M_{2^{n}}(K) \stackrel{f_{n}}{\hookrightarrow} M_{2^{n+1}}(K) \stackrel{f_{n+1}}{\hookrightarrow} \ldots,
$$

where $f_{n}: M_{2^{n}}(K) \hookrightarrow M_{2^{n+1}}(K)$ is the block-diagonal embedding defined by $f_{n}(A)=\left(\begin{array}{cc}A & 0 \\ 0 & A\end{array}\right)$.

$\mathcal{D}$ will be the class of all modules that have no maximal submodules. Clearly $\mathcal{D}$ is closed under direct summands and extensions. Moreover, $A$ is a hereditary ring, and $\operatorname{Ext}_{A}^{1}(M, N) \neq 0$ whenever $M, N$ are non-zero finitely generated and $M$ is non-projective by [11, Lemma 3.2 and Proposition 3.3]. Baer's Criterion then yields that $\mathcal{D}$ contains all injective modules.

We claim that $\mathcal{D}$ is closed under direct products. Note that each simple module is slender by [10, Lemma 3.7]. Let $\kappa$ be a (non- $\omega$-measurable) cardinal and consider a sequence $\left(D_{\alpha} \mid \alpha<\kappa\right)$ of elements of $\mathcal{D}$. By [3, Corollary III.3.4], any non-zero homomorphism from $\prod_{\alpha<\kappa} D_{\alpha}$ to a simple module $S$ is necessarily non-zero on the direct sum $\bigoplus_{\alpha<\kappa} D_{\alpha}$, and hence on some $D_{\alpha}$, in contradiction with $D_{\alpha} \in \mathcal{D}$. This proves our claim.

Finally assume that $\mathcal{D}=\mathcal{C}^{\perp}$ for a class of modules $\mathcal{C}$. Since $\mathcal{D}$ is closed under direct sums and $A$ is hereditary, the class $\mathcal{D}$ is 1 -tilting by [6, Corollary 6.1.7], so $\mathcal{D}=\operatorname{Mod}-A$ by $[6$, Corollary 6.2.4], a contradiction.

In the next few lemmas, we will work with particular chains $\left(Y_{\alpha} \mid \alpha \leq \sigma\right)$ consisting of submodules of a module $Y$ (where $\sigma$ is an ordinal).

A chain $\left(Y_{\alpha} \mid \alpha \leq \sigma\right)$ is called a strictly increasing continuous chain of submodules of $Y$ provided that $Y_{0}=0, Y_{\alpha} \subsetneq Y_{\alpha+1}$ for each $\alpha<\sigma, Y_{\alpha}=\bigcup_{\beta<\alpha} Y_{\beta}$ for each limit ordinal $\alpha \leq \sigma$, and $Y_{\sigma}=M$.

Dually, $\left(Y_{\alpha} \mid \alpha \leq \sigma\right)$ is a strictly decreasing continuous chain of submodules of $Y$ provided that $Y_{0}=Y, Y_{\alpha} \supsetneq Y_{\alpha+1}$ for each $\alpha<\sigma, Y_{\alpha}=\bigcap_{\beta<\alpha} Y_{\beta}$ for each limit ordinal $\alpha \leq \sigma$, and $Y_{\sigma}=0$.

The following result was proved in [12, Theorem 2] for finite rank torsion-free abelian groups. Here we simplify our notation by writing ${ }^{\circ} X$ instead of ${ }^{\circ}\{X\}$ for a module $X$, and similarly for the other annihilator classes.

Lemma 3. Let $A$ be a ring, and $X, Y$ be non-zero modules. Then the following conditions are equivalent:

a) ${ }^{\circ} X \subseteq{ }^{\circ} Y$ (that is, $\left.Y \in\left({ }^{\circ} X\right)^{\circ}\right)$.

b) There exist a strictly decreasing continuous chain $\left(Y_{\alpha} \mid \alpha \leq \sigma\right)$ of submodules of $Y$, and A-homomorphisms $\varphi_{\alpha}: Y_{\alpha} \rightarrow X(\alpha<\sigma)$, such that $Y_{\alpha+1}=\operatorname{Ker}\left(\varphi_{\alpha}\right)$ for all $\alpha<\sigma$.

Moreover, if $X=\bigoplus_{\gamma<\kappa} X_{\gamma}$ is any direct sum decomposition of $X$, then we can choose each homomorphism $\varphi_{\alpha}$ so that $\operatorname{Im}\left(\varphi_{\alpha}\right) \subseteq X_{\gamma}$ for some $\gamma<\kappa$.

Proof. a) $\Rightarrow$ b) We construct the chain $\left(Y_{\alpha} \mid \alpha \leq \sigma\right)$ by induction on $\alpha$. Let $Y_{0}=Y$. Assume $Y_{\alpha}$ is defined and $Y_{\alpha} \neq 0$. Then $\operatorname{Hom}_{A}\left(Y_{\alpha}, Y\right) \neq 0$, so there exists a nonzero homomorphism $\varphi_{\alpha}: Y_{\alpha} \rightarrow X$. Without loss of generality, we can suppose that $\operatorname{Im}\left(\varphi_{\alpha}\right) \subseteq X_{\gamma}$ for some $\gamma<\kappa$, and put $Y_{\alpha+1}=\operatorname{Ker}\left(\varphi_{\alpha}\right) \subsetneq Y_{\alpha}$. If $\alpha$ is a limit ordinal, we define $Y_{\alpha}=\bigcap_{\beta<\alpha} Y_{\beta}$. Since the $Y_{\alpha}$ s form a strictly decreasing chain, our construction must stop at some $\sigma$, hence $Y_{\sigma}=0$.

b) $\Rightarrow$ a) Suppose that $\varphi: U \rightarrow Y$ is a non-zero homomorphism. Then there is a least index $\alpha<\sigma$ such that $\operatorname{Im}(\varphi) \subseteq Y_{\alpha}$. So $\operatorname{Im}(\varphi) \nsubseteq Y_{\alpha+1}$, and $0 \neq \varphi_{\alpha} \varphi: U \rightarrow X$. Therefore ${ }^{\circ} X \subseteq{ }^{\circ} Y$. 
Of course, the strictly decreasing chain in condition b) is always finite when $Y$ is artinian. But if $Y$ is only assumed noetherian then an infinite chain is needed in general in condition b) even if $X$ has finite length.

For a simple example, consider the case of the abelian groups $X=\mathbb{Z}_{p}=\mathbb{Z} / p \mathbb{Z}$ ( $p$ a prime integer) and $Y=\mathbb{Z}$. Then ${ }^{\circ} X$ is the class of all groups containing no maximal subgroup of index $p$, and ${ }^{\circ} Y$ the (larger) class of all groups having no non-zero free summands. Notice that there exists exactly one chain satisfying condition b), namely $Y_{n}=\mathbb{Z} p^{n}$ for $n<\omega$ and $Y_{\omega}=0$.

We could have expressed condition b) of Lemma 3 simply in terms of the existence of a strictly decreasing continuous chain of submodules of $Y,\left(Y_{\alpha} \mid \alpha \leq \sigma\right)$, such that $Y_{\alpha} / Y_{\alpha+1}$ is isomorphic to a submodule of $X$ for each $\alpha<\sigma$.

The dual result, concerning the annihilator classes of the form $X^{\circ}$, then reads as follows:

Lemma 4. Let $A$ be a ring, and $X, Y$ be non-zero modules. Then the following conditions are equivalent:

a) $X^{\circ} \subseteq Y^{\circ}$ (that is, $Y \in{ }^{\circ}\left(X^{\circ}\right)$ ).

b) There exist a strictly increasing continuous chain $\left(Y_{\alpha} \mid \alpha \leq \sigma\right)$ of submodules of $Y$ such that $Y_{\alpha+1} / Y_{\alpha}$ is a homomorphic image of $X$ for each $\alpha<\sigma$.

Moreover, if $X=\bigoplus_{\gamma<\kappa} X_{\gamma}$ is any direct sum decomposition of $X$, then in condition $b)$, we can assume, without loss of generality, that for each $\alpha<\sigma$, the module $Y_{\alpha+1} / Y_{\alpha}$ is a homomorphic image of $X_{\gamma}$ for some $\gamma<\kappa$.

Proof. a) $\Rightarrow$ b) We construct the chain $\left(Y_{\alpha} \mid \alpha \leq \sigma\right)$ by induction on $\alpha$. Let $Y_{0}=0$. Assume $Y_{\alpha}$ is defined and $Y_{\alpha} \neq Y$. Then $\operatorname{Hom}_{A}\left(Y, Y / Y_{\alpha}\right) \neq 0$, so there exists a non-zero homomorphism $f: X \rightarrow Y / Y_{\alpha}$ (If $X=\bigoplus_{\beta<\kappa} X_{\beta}$, we replace $f$ by its non-zero restriction to some $X_{\beta}$.) We take $Y_{\alpha+1} \subseteq Y$ such that $Y_{\alpha} \subsetneq Y_{\alpha+1}$ and $Y_{\alpha+1} / Y_{\alpha}=\operatorname{Im}(f)$. For a limit ordinal $\alpha$, we define $Y_{\alpha}=\bigcup_{\beta<\alpha} Y_{\beta}$. Clearly, the construction stops at some ordinal $\sigma$, so $Y_{\sigma}=Y$.

b) $\Rightarrow$ a) If $\varphi: Y \rightarrow U$ is a non-zero homomorphism, then there is a least index $\beta<\sigma$ such that $\operatorname{Ker}(\varphi) \nsupseteq Y_{\beta}$. Then $\beta=\alpha+1$ is a non-limit ordinal, $\operatorname{Ker}(\varphi) \supseteq Y_{\alpha}$, and $\varphi$ has a factorization through the canonical projection $\pi: Y \rightarrow Y / Y_{\alpha}, \varphi=\psi \pi$ where $\psi: Y / Y_{\alpha} \rightarrow U$ is non-zero when restricted to $Y_{\beta} / Y_{\alpha}$. By assumption, there is an epimorhism $f: X \rightarrow Y_{\beta} / Y_{\alpha}$, hence $0 \neq \psi f: X \rightarrow U$.

The strictly increasing chain in condition b) of Lemma 4 must be finite in case $Y$ is noetherian. However, if $Y$ is only assumed artinian then an infinite chain is needed in general even if $X$ has finite length. Again, consider the case of abelian groups, $X=\mathbb{Z}_{p}$ ( $p$ a prime integer) and $Y=\mathbb{Z}_{p^{\infty}}$ (the Prüfer group). Then $X^{\circ}$ is the class of all groups with trivial $p$-torsion part while $Y^{\circ}$ is the (larger) class of all groups with no direct summand isomorphic to $Y$. The only chain satisfying condition b) of Lemma 4 is the (unique) composition series of $\mathbb{Z}_{p^{\infty}}$.

Now we turn to the annihilator classes of the form $X^{\perp}$ and generalize $[8$, Lemma 5.2]. We will call a module $M$ torsionless provided that $M$ embeds into a free module.

Lemma 5. Let $A$ be a ring, and $X, Y$ be non-zero modules. Consider the following conditions:

a) $X^{\perp} \subseteq Y^{\perp}$ (that is, $Y \in{ }^{\perp}\left(X^{\perp}\right)$ ). 
b) $Y$ is a direct summand in a module $Z$ such that $Z$ possesses a strictly increasing continuous chain of submodules $\left(Z_{\alpha} \mid \alpha \leq \rho\right)$ with $Z_{1}$ a free module, and $Z_{\alpha+1} / Z_{\alpha} \cong X$ for each $0<\alpha<\rho$.

c) There exist a strictly increasing continuous chain $\left(Y_{\alpha} \mid \alpha \leq \sigma\right)$ of submodules of $Y$ and A-homomorphisms $\varphi_{\alpha}: Y_{\alpha+1} \rightarrow X(0<\alpha<\sigma)$ such that $Y_{\alpha}=\operatorname{Ker}\left(\varphi_{\alpha}\right)$ for all $0<\alpha<\sigma$. Moreover, either $Y_{1}$ is torsionless or $Y_{1}$ embeds into $X$.

Then a) is equivalent to $b$ ), and it implies c).

Moreover, if $X=\bigoplus_{\gamma<\kappa} X_{\gamma}$ is any direct sum decomposition of $X$, then we can choose each homomorphism $\varphi_{\alpha}(0<\alpha<\sigma)$ in c) so that $\operatorname{Im}\left(\varphi_{\alpha}\right) \subseteq X_{\gamma}$ for some $\gamma<\kappa$.

Conversely, c) implies b) if and only if $X^{\perp}$ consists of modules of injective dimension $\leq 1$.

Proof. The equivalence of a) and b) is well-known (see e.g. [6, 3.2.3]).

Assume b). Let $Y_{\alpha}=Y \cap Z_{\alpha}$ for each $\alpha \leq \rho$. Then $Y_{0}=0, Y_{1}$ is torsionless (but possibly zero), $Y_{\alpha} \subseteq Y_{\alpha+1}$, and $Y_{\alpha+1} / Y_{\alpha} \cong\left(Z_{\alpha}+Y_{\alpha+1}\right) / Z_{\alpha} \subseteq Z_{\alpha+1} / Z_{\alpha} \cong X$ for each $0<\alpha<\rho$. Moreover, $Y_{\alpha}=Y \cap Z_{\alpha}=\bigcup_{\beta<\alpha} Y_{\beta}$ for each limit ordinal $\alpha \leq \rho$, and $Y_{\rho}=Y$. Removing possible repetitions from the chain $\left(Y_{\alpha} \mid \alpha \leq \rho\right)$, we obtain the required strictly increasing chain as in c).

If $X=\bigoplus_{\gamma<\kappa} X_{\gamma}$ then we can refine the original chain $\left(Z_{\alpha} \mid \alpha \leq \rho\right)$ so that each consecutive factor $Z_{\alpha+1} / Z_{\alpha}$ is isomorphic to some $X_{\gamma}$, and then proceed as in the previous paragraph. Then for each $0<\alpha<\sigma$ there is some $\gamma<\kappa$ such that $\operatorname{Im}\left(\varphi_{\alpha}\right) \subseteq X_{\gamma}$.

Assume c). For each $\alpha<\sigma$, the $A$-homomorphism $\varphi_{\alpha}$ yields an embedding $Y_{\alpha+1} / Y_{\alpha} \hookrightarrow X$. If $X^{\perp}$ consist of modules of injective dimension $\leq 1$, then $\operatorname{Ext}_{A}^{1}\left(Y_{\alpha+1} / Y_{\alpha}, M\right)=0$ for each $M \in X^{\perp}$, and a) follows by the Eklof Lemma [6, 3.1.2].

Conversely, if $X^{\perp}$ contains a module $M$ of injective dimension $>1$, then by Baer's Criterion, there is a right ideal $Y$ of $A$ such that $\operatorname{Ext}_{A}^{1}(Y, M) \neq 0$. Since $Y$ is torsionless, condition c) of Lemma 5 holds for $\sigma=1$, but a) fails since $M \in$ $X^{\perp} \backslash Y^{\perp}$.

Corollary 6. $A$ ring $A$ is right hereditary if and only the conditions b) and c) in Lemma 5 are equivalent for all right $A$-modules $X$ and $Y$.

Proof. If $A$ is not hereditary and $X$ is a projective right $A$-module then the class $X^{\perp}=\operatorname{Mod}-A$ contains an element of injective dimension at least 2 . The last part of Lemma 5 shows that, under this hypothesis, the conditions b) and c) are not equivalent.

The condition b) of Lemma 3 is quite close to condition c) of Lemma 5 in case $Y$ is both artinian and noetherian, that is, when $Y$ has finite length:

Theorem 7. Let $A$ be a ring, and $X, Y$ be non-zero modules. Consider the following conditions:
a) ${ }^{\circ} X \subseteq{ }^{\circ} Y$;

b) $X^{\perp} \subseteq Y^{\perp}$.

Then a) implies b) in case $Y$ is artinian and $X^{\perp}$ consists of modules of injective dimension $\leq 1$. 
Conversely, b) implies a) in case $Y$ is noetherian and $Y$ has no non-zero torsionless submodules.

In particular, a) is equivalent to b) in case $A$ is right hereditary, $Y$ has finite length and contains no non-zero projective submodules.

Clearly, the binary relations $\preceq$ and $\sqsubseteq$ defined on the set of all finite length modules by $X \preceq Y$ if and only if ${ }^{\circ} X \subseteq{ }^{\circ} Y$, and by $X \sqsubseteq Y$ if and only if $X^{\perp} \subseteq Y^{\perp}$, are preorder relations. We can now characterize the hereditary rings over which these relations coincide:

Theorem 8. The following are equivalent for a right hereditary ring A:

a) Either $A$ is simple artinian, or $\operatorname{Soc}(A)=0$.

b) ${ }^{\circ} X \subseteq{ }^{\circ} Y$ if and only if $X^{\perp} \subseteq Y^{\perp}$, for all non-zero finite length modules $X$ and $Y$.

c) ${ }^{\circ} X={ }^{\circ} Y$ if and only if $X^{\perp}=Y^{\perp}$, for all non-zero finite length modules $X$ and $Y$.

Proof. a $\Rightarrow \mathrm{b}$ ) If $A$ is simple artinian then b) is clear. If $\operatorname{Soc}(A)=0$, then there are no simple projective modules and b) holds by the final claim of Theorem 7 .

b) $\Rightarrow$ c) is obvious.

c) $\Rightarrow$ a) Suppose that $S$ and $T$ are non-isomorphic simple modules such that $S$ is projective. Then $(S \oplus T)^{\perp}=(T \oplus T)^{\perp}$, hence ${ }^{\circ}(S \oplus T)={ }^{\circ}(T \oplus T)$. This gives $\operatorname{Hom}(S, T) \neq 0$, a contradiction. So either all simple modules are isomorphic and projective, or there are no projective simple modules.

If all simple modules are isomorphic and projective, then each maximal right ideal $M$ is a direct summand in $A$, so the socle of $A$ is not contained in $M$, hence $A$ is simple artinian.

If there are no projective simple modules then $\operatorname{Soc}(A)=0$ because $A$ is right hereditary.

Our next result is a variant of the equivalence a) $\Leftrightarrow b$ ) of Theorem 7 that restricts condition a) to finite length modules with no projective direct summands:

Proposition 9. Let $A$ be a right hereditary ring and $Y$ a module of finite length. The following are equivalent:

a) $X^{\perp} \subseteq Y^{\perp}$

b) ${ }^{\circ} X \cap \mathcal{C} \subseteq{ }^{\circ} Y \cap \mathcal{C}$, where $\mathcal{C}$ is the class of all finite length modules which have no non-zero projective direct summands.

Proof. a) $\Rightarrow$ b) If $Y$ is projective then clearly ${ }^{\circ} X \cap \mathcal{C} \subseteq{ }^{\circ} Y \cap \mathcal{C}=\mathcal{C}$.

Suppose that $Y$ is not projective. By Lemma 5 there are a finite strictly increasing continuous chain $\left(Y_{\alpha} \mid \alpha \leq n\right)$ of submodules of $Y$ and $A$-homomorphisms $\varphi_{\alpha}: Y_{\alpha+1} \rightarrow X(0<\alpha<n)$ such that $Y_{\alpha}=\operatorname{Ker}\left(\varphi_{\alpha}\right)$ for all $0<\alpha<n$ and $Y_{1}$ is either projective or embeds into $X$. In the latter case, let $\varphi_{0}$ denote an embedding of $Y_{1}$ into $X$.

Let $Z \in \mathcal{C}$ and suppose that $Z \notin{ }^{\circ} Y$, so there is a non-zero homomorphism $f: Z \rightarrow Y$. Observe that $\operatorname{Im}(f) \nsubseteq Y_{1}$ in case $Y_{1}$ is projective. There is an index $0<\alpha \leq n$ such that $\operatorname{Im}(f) \subseteq Y_{\alpha}$ and $\operatorname{Im}(f) \nsubseteq Y_{\alpha-1}$. Then $g=\varphi_{\alpha-1} f$ gives a non-zero homomorphism from $Z$ into $X$, and $Z \notin^{\circ} X$.

b) $\Rightarrow$ a) There is nothing to prove if $Y$ is projective.

Suppose that $Y$ is not projective. Since $Y$ has finite length, $Y$ has a decomposition $Y=P_{0} \oplus C_{0}$ such that $P_{0}$ is projective and $0 \neq C_{0} \in \mathcal{C}$. By assumption 
there exists a non-zero homomorphism $f_{0}: C_{0} \rightarrow X$. Let $Z_{0}=\operatorname{Ker}\left(f_{0}\right) \subsetneq C_{0}$. If $Z_{0}$ is not projective, we decompose it as $Z_{0}=P_{1} \oplus C_{1}$ where $P_{1}$ projective and $0 \neq C_{1} \in \mathcal{C}$. Then there exists a non-zero homomorphism $f_{1}: C_{1} \rightarrow X$ and we define $Z_{1}=\operatorname{Ker}\left(f_{1}\right) \subsetneq C_{1}$. Proceeding in this way, we obtain a decreasing chain $Y \supseteq C_{0} \supsetneq Z_{0} \supseteq C_{1} \supsetneq Z_{1} \supseteq \ldots$ and projective modules $P_{0}, \ldots, P_{n}$ such that $Z_{n-1}=P_{n} \oplus C_{n}$. Since $Y$ has finite length, there is an $n<\omega$ such that the construction stops, i.e., $Z_{n}$ is projective.

We define an increasing chain of submodules of $Y$ as follows: $Y_{0}=0, Y_{1}=$ $P_{0} \oplus P_{1} \oplus \cdots \oplus P_{n} \oplus Z_{n}, Y_{2}=Y_{1}+C_{n}, \ldots, Y_{n+1}=Y_{1}+C_{1}, Y_{n+2}=Y_{1}+C_{0}=Y$. Then $Y_{1}$ is projective, and we have

$$
\begin{gathered}
Y_{k+1} / Y_{k}=\left(Y_{1}+C_{n-k+1}\right) /\left(Y_{1}+C_{n-k+2}\right) \cong \\
C_{n-k+1} /\left(\left(Y_{1}+C_{n-k+2}\right) \cap C_{n-k+1}\right)=C_{n-k+1} / Z_{n-k+1}
\end{gathered}
$$

because in the construction above, $Y_{1}+C_{n-k+2}=P_{0}+\cdots+P_{n-k+1}+Z_{n-k+1}$, $Z_{n-k+1} \subsetneq C_{n-k+1}$, and $\left(P_{0}+\cdots+P_{n-k+1}\right) \cap C_{n-k+1}=0$ by construction, so $Y_{k+1} / Y_{k}$ is isomorphic to a submodule of $X$ for each $1 \leq k \leq n+1$. By Lemma 5 we conclude that $X^{\perp} \subseteq Y^{\perp}$.

Concerning the dual classes $X^{\circ}$ and ${ }^{\perp} X$, the reader might expect a dualization of Lemma 5. There is no such result available. In other words, there is no handy description of the class $\left({ }^{\perp} X\right)^{\perp}$ for an arbitrary module $X$ (see [6, §3.3] for more details).

We will see in Example 12 that the dual of the implication $b) \Rightarrow a$ ) in Theorem 7 fails in general. However, the dual of the implication $a) \Rightarrow b$ ) does hold:

Lemma 10. Let $A$ be a ring, and $X, Y$ be non-zero modules such that $Y$ is noetherian and ${ }^{\perp} X$ consists of modules of projective dimension $\leq 1$.

Then $X^{\circ} \subseteq Y^{\circ}$ implies ${ }^{\perp} X \subseteq{ }^{\perp} Y$.

Proof. By Lemma 4, there exist $0<m<\omega$ and a strictly increasing continuous chain $\left(Y_{n} \mid n \leq m\right)$ of submodules of $Y$ such that $Y_{n+1} / Y_{n}$ is a homomorphic image of $X$ for each $n<m$. Let $M \in{ }^{\perp} X$. Then $M$ has projective dimension $\leq 1$, so $\operatorname{Ext}_{A}^{1}\left(M, Y_{n+1} / Y_{n}\right)=0$ for each $n<m$, and by induction on $n$, we conclude that $M \in{ }^{\perp} Y$.

The equivalence a) $\Leftrightarrow \mathrm{b}$ ) in Theorem 7 holds in particular for all hereditary artin algebras $A$ (and all finitely generated modules $Y$ containing no indecomposable projective submodules). For these algebras, the dual result does hold. In order to prove this we need to recall that if $A$ is a hereditary artin algebra and $\tau=\mathrm{DTr}$ is the Auslander-Reiten translation in the category of all finitely presented modules, then by the Auslander-Reiten formula we get an isomorphism $\operatorname{Hom}_{A}(X, \tau N) \cong$ $\operatorname{DExt}_{A}^{1}(N, X)$ for each finitely presented $A$-module $N$ and each $A$-module $X$ (see e.g. [8, Lemma 1.1]).

Proposition 11. Let $A$ be a hereditary artin algebra. Let $X, Y$ be non-zero modules such that $Y$ has finite length and $Y$ has no indecomposable injective factor-modules. Then $X^{\circ} \subseteq Y^{\circ}$ if and only if ${ }^{\perp} X \subseteq{ }^{\perp} Y$.

Proof. The direct implication holds by Lemma 10.

For the converse, assume that ${ }^{\perp} X \subseteq{ }^{\perp} Y$. We have to show that $X^{\circ} \subseteq Y^{\circ}$. Since $Y$ is finitely presented, $Y^{\circ}$ is a torsion-free class closed under direct limits, so it 
suffices to prove the implication

$$
\operatorname{Hom}_{A}(X, M)=0 \Rightarrow \operatorname{Hom}_{A}(Y, M)=0
$$

for each finitely generated module $M$.

By the assumption on $Y$, if this implication fails, then it fails for a finitely generated module $M$ with no indecomposable injective direct summands. So it suffices to prove the implication when $M$ is finitely generated, indecomposable and not injective. Then $M=\tau N$ for a finitely generated indecomposable module $N$, so $\operatorname{Hom}_{A}(X, M)=\operatorname{Hom}_{A}(X, \tau N)=0$ implies $\operatorname{Ext}_{A}(N, X)=0$ by the AuslanderReiten formula, hence $\operatorname{Ext}_{A}(N, Y)=0$ by assumption. Then also $\operatorname{Hom}_{A}(Y, M)=$ $\operatorname{Hom}_{A}(Y, \tau N)=0$, q.e.d.

The reverse implication of Proposition 11 will clearly fail for all injective simple modules $X, Y$ such that $X \nRightarrow Y$. We finish this section by an example showing that it is not possible to extend Proposition 11 to general hereditary rings, even if we restrict ourselves only to non-injective simple modules:

Example 12. Let $A$ be a simple countable, but not artinian, von Neumann regular ring (so $A$ is as in Example 2, but we moreover require the field $K$ to be countable). By [11, Proposition 6.3], any representative set simp- $A$ of all simple modules has $2^{\omega}$ elements, and all of them are non-injective. The condition $X^{\circ} \subseteq Y^{\circ}$ clearly fails for all $X \neq Y \in \operatorname{simp}-A$. However, assuming $\mathrm{V}=\mathrm{L}$, for each module $X$ of finite length, the class ${ }^{\perp} X$ coincides with the class of all projective modules by [11, Corollary 3.19]. In particular, the condition ${ }^{\perp} X \subseteq{ }^{\perp} Y$ holds for all $X, Y \in \operatorname{simp}-A$.

\section{Modules of Finite Length and their annihilator Classes}

Now we turn to finite length modules that are direct sums of bricks.

Recall that a module $X$ is a brick if $\operatorname{End}_{A}(X)$ is a division ring. Notice that any brick is indecomposable.

Theorem 13. Let $A$ be a ring, and $X, Y$ be non-zero modules of finite length. Assume that $X=\bigoplus_{i=1}^{m} X_{i}$ is a direct sum of bricks such that $\operatorname{Hom}\left(X_{i}, X_{j}\right)=0$ if $i \neq j$. Then the following are equivalent:

a) $X \cong Y$;

b) ${ }^{\circ} X={ }^{\circ} Y$ and $\lg (Y) \leq \lg (X)$;

c) ${ }^{\circ} X={ }^{\circ} Y$ and $\lg (X)=\lg (Y)$;

d) ${ }^{\circ} X={ }^{\circ} Y$ and $Y=\bigoplus_{i=1}^{n} Y_{i}$ is a direct sum of bricks such that $\operatorname{Hom}\left(Y_{i}, Y_{j}\right)=$ 0 for all $i \neq j$.

Proof. The implications a) $\Rightarrow \mathrm{d}$ ) and $\mathrm{c}) \Rightarrow \mathrm{b}$ ) are trivial.

b) $\Rightarrow$ a) By Lemma 3 there are a chain of submodules $Y=Z_{0} \supsetneq Z_{1} \supsetneq \cdots \supsetneq Z_{k}=$ 0 and homomorphisms $\varphi_{i}: Z_{i} \rightarrow X$ such that $\operatorname{Ker}\left(\varphi_{i}\right)=Z_{i+1}$, and for each $i<k$ there is $j \in\{1, \ldots, m\}$ with $\operatorname{Im}\left(\varphi_{i}\right) \subseteq X_{j}$.

Let $j \in\{1, \ldots, m\}$. Since $\operatorname{Hom}\left(X_{j}, X\right) \neq 0$, there exists a non-zero homomorphism $f: X_{j} \rightarrow Y$. Then there is an index $i<k$ such that $\operatorname{Im}(f) \subseteq Z_{i}$ and $\operatorname{Im}(f) \nsubseteq Z_{i+1}$, hence $\varphi_{i} f \neq 0$. Since $\operatorname{Hom}\left(X_{j}, X_{s}\right)=0$ for all $s \neq j$, we have $0 \neq \operatorname{Im}\left(\varphi_{i} f\right) \subseteq X_{j}$, and $\operatorname{Im}\left(\varphi_{i}\right) \subseteq X_{j}$. If $\pi_{j}: X \rightarrow X_{j}$ is the canonical projection, then $\pi_{j} \varphi_{i} f$ is an isomorphism because $X_{j}$ is a brick. It follows that $f: X_{j} \rightarrow Z_{i}$ is a split monomorphism, and

$$
Z_{i}=f\left(X_{j}\right) \oplus \operatorname{Ker}\left(\pi_{j} \varphi_{i}\right)=f\left(X_{j}\right) \oplus \operatorname{Ker}\left(\varphi_{i}\right) \cong X_{j} \oplus \operatorname{Ker}\left(\varphi_{i}\right) .
$$


Since $\operatorname{Ker}\left(\varphi_{i}\right)=Z_{i+1}$, there is a subset $I=\left\{i_{1}, \ldots, i_{m}\right\} \subseteq\{0, \ldots, k-1\}$ such that $Z_{i_{j}+1}$ is a direct summand of $Z_{i_{j}}$ with a complement isomorphic to $X_{j}$, for all $j \in\{1, \ldots, m\}$. In particular, $\lg (X) \leq \lg (Y)$.

But $\lg (X) \geq \lg (Y)$ by assumption, so $I=\{0, \ldots, k-1\}$ and $Y \cong \bigoplus_{i=1}^{m} X_{i}=X$.

d) $\Rightarrow$ c) As in the proof of b) $\Rightarrow$ a), the inclusion ${ }^{\circ} X \subseteq{ }^{\circ} Y$ implies $\lg (X) \leq \lg (Y)$. Swapping the roles of $X$ and $Y$, we obtain that $\lg (Y) \leq \lg (X)$.

We also have the dual result:

Theorem 14. Let $A, X$ and $Y$ be as in Theorem 13. Then the following are equivalent:
a) $X \cong Y$;
b) $X^{\circ}=Y^{\circ}$ and $\lg (Y) \leq \lg (X)$;
c) $X^{\circ}=Y^{\circ}$ and $\lg (X)=\lg (Y)$;
d) $X^{\circ}=Y^{\circ}$ and $Y=\bigoplus_{i=1}^{n} Y_{i}$ is a direct sum of bricks such that $\operatorname{Hom}\left(Y_{i}, Y_{j}\right)=$ 0 for all $i \neq j$.

Proof. We only give the proof for the implication $b) \Rightarrow a$ ), the rest is easy. By Lemma 4 there is a chain of submodules $0=Y_{0} \subsetneq Y_{1} \subsetneq \cdots \subsetneq Y_{k}=Y$ such that $Y_{i+1} / Y_{i}$ is a homomorphic image of $X_{j}$ for some $j \in\{1, \ldots, m\}$.

Let $j \in\{1, \ldots, m\}$. Since $\operatorname{Hom}\left(X, X_{j}\right) \neq 0$, also $\operatorname{Hom}\left(Y, X_{j}\right) \neq 0$, so there exist $i<k$ and a non-zero homomorphism $f: Y_{i+1} / Y_{i} \rightarrow X_{j}$. Since $\operatorname{Hom}\left(X_{s}, X_{j}\right)=0$ for all $s \neq j$, there is an epimorphism $\pi: X_{j} \rightarrow Y_{i+1} / Y_{i}$. But $X_{j}$ is a brick, so $\pi$ is an isomorphism. In particular, $\lg (X) \leq \lg (Y)$. The assumption of $\lg (X) \geq \lg (Y)$ then implies that $k=m$, and there is a permutation $p \in S_{m}$ such that $Y_{i+1} / Y_{i} \cong X_{p(i)}$ for all $i<m$. Without loss of generality, we will assume that $p=$ id.

Since $\operatorname{Hom}\left(X, Y_{1}\right) \neq 0$, there exists $0 \neq f \in \operatorname{Hom}\left(Y, Y_{1}\right)$. As $\operatorname{Hom}\left(X_{j}, X_{0}\right)=0$ for all $j \neq 0$, we have $f \nmid Y_{1} \neq 0$. But $Y_{1}$ is a brick, so the inclusion $Y_{1} \subseteq Y$ splits, and $Y=Y_{1} \oplus Y^{\prime}$ where $Y^{\prime}$ possesses a chain $0=Y_{0}^{\prime} \subsetneq Y_{1}^{\prime} \subsetneq \cdots \subsetneq Y_{m-1}^{\prime}=Y^{\prime}$ with $Y_{i+1}^{\prime} / Y_{i}^{\prime} \cong X_{i+1}$ for all $i<m-1$.

In particular, $Y_{1}^{\prime} \cong X_{1}$, so $\operatorname{Hom}\left(X, Y_{1}^{\prime}\right) \neq 0$. Then $\operatorname{Hom}\left(Y, Y_{1}^{\prime}\right) \neq 0$, and also $\operatorname{Hom}\left(Y^{\prime}, Y_{1}^{\prime}\right) \neq 0$ because $\operatorname{Hom}\left(X_{0}, X_{1}\right)=0$. Consider $0 \neq f^{\prime} \in \operatorname{Hom}\left(Y^{\prime}, Y_{1}^{\prime}\right)$. Then $f^{\prime}\left\lceil Y_{1}^{\prime} \neq 0\right.$. Since $Y_{1}^{\prime}$ is a brick, the inclusion $Y_{1}^{\prime} \subseteq Y^{\prime}$ splits, giving a decomposition $Y^{\prime}=Y_{1}^{\prime} \oplus Y^{\prime \prime}$. Proceeding in this way, we obtain a direct sum decomposition of $Y$ showing that $Y \cong \bigoplus_{i=1}^{m} X_{i}=X$.

There is a similar result for the annihilator classes of the form $X^{\perp}$ over hereditary rings:

Theorem 15. Let $A$ be a right hereditary ring, and $X, Y$ be non-zero modules of finite length. Assume that $X=\bigoplus_{i=1}^{m} X_{i}$ is a direct sum of non-projective bricks such that $\operatorname{Hom}\left(X_{i}, X_{j}\right)=0$ if $i \neq j$. Then the following are equivalent:
a) $X \cong Y$;
b) $X^{\perp}=Y^{\perp}$ and $\lg (Y) \leq \lg (X)$;
c) $X^{\perp}=Y^{\perp}$ and $\lg (X)=\lg (Y)$;
d) $X^{\perp}=Y^{\perp}$ and $Y=\bigoplus_{i=1}^{n} Y_{i}$ is a direct sum of bricks such that $\operatorname{Hom}\left(Y_{i}, Y_{j}\right)=$ 0 for all $i \neq j$.

Proof. This is again a variation of the proof of Theorem 13, so we only indicate the necessary changes: 
- We use Lemma 5 in place of Lemma 3, so the module $Z_{k}$ can be non-zero, but it is always projective.

- If $j \in\{1, \ldots, m\}$, using $Y^{\perp}=X^{\perp} \subseteq X_{j}^{\perp}$ and Proposition 9, we deduce that there exists a non-zero homomorphism $f: X_{j} \rightarrow Y$. Since the brick $X_{j}$ is not projective, $\operatorname{Im}(f) \nsubseteq Z_{k}$. Then there exists an index $i_{j} \in\{0, \ldots, k-1\}$ such that $Z_{i_{j}+1}$ is a direct summand of $Z_{i_{j}}$ with a complement isomorphic to $X_{j}$.

- In the last part, from the equalities of lengths we obtain that $Z_{k}=0$.

The rest of the proof is the same as for Theorem 13 .

Remark 16. By Example 12, assuming $V=L$, there exist a hereditary $\operatorname{ring} A$ and non-isomorphic non-injective simple modules (hence bricks) $S$ and $T$ such that ${ }^{\perp} S={ }^{\perp} T$. So the dual of Theorem 15 cannot be proved in ZFC.

Next we list without proof several elementary properties of the annihilator classes of the form ${ }^{\circ} X$ :

Lemma 17. Let $A$ be a ring, and $X, Y$, and $Z$ be modules.

a) ${ }^{\circ}(X \oplus Y)={ }^{\circ} X \cap{ }^{\circ} Y$;

b) If $0 \rightarrow X \rightarrow Y \rightarrow Z \rightarrow 0$ is a short exact sequence then ${ }^{\circ}(X \oplus Z) \subseteq{ }^{\circ} Y \subseteq$ ${ }^{\circ} X$;

c) If $f \in \operatorname{End}(X)$ and $K=\operatorname{Ker}(f)$ then ${ }^{\circ} X={ }^{\circ}(K \oplus X / K)$. If moreover $f^{2}=0$ then also ${ }^{\circ} X={ }^{\circ} K$.

The following lemma gives a way of replacing finite length modules by direct sums of bricks without changing the annihilator class:

Lemma 18. Let $A$ be a ring and $Y$ be a non-zero module of finite length. Then there exists a chain of submodules $Y=Y_{0} \supsetneq Y_{1} \supsetneq \cdots \supsetneq Y_{k}$ such that

i) ${ }^{\circ} Y={ }^{\circ} Y_{1}=\cdots={ }^{\circ} Y_{k}$,

ii) $Y_{k}=\bigoplus_{i=1}^{m} Z_{i}$ where each $Z_{i}$ is a brick such that $\operatorname{Hom}\left(Z_{i}, Z_{j}\right)=0$ for all $i \neq j$

iii) $Y_{j} / Y_{j+1}$ embeds into $Y_{j}$ for all $j \in\{0, \ldots, k-1\}$.

Proof. Suppose that $Y$ has a non-zero nilpotent endomorphism. Let $Y_{0}=Y$, and take $0 \neq f_{0} \in \operatorname{End}_{A}\left(Y_{0}\right)$ such that $f_{0}^{2}=0$. Let $Y_{1}=\operatorname{Ker}\left(f_{0}\right) \subsetneq Y_{0}$. If $Y_{1}$ has a non-zero nilpotent endomorphism, take $0 \neq f_{1} \in \operatorname{End}_{A}\left(Y_{1}\right)$ such that $f_{1}^{2}=0$ and let $Y_{2}=\operatorname{Ker}\left(f_{1}\right) \subsetneq Y_{1}$.

Since $Y$ is of finite length, there is a least $k$ such that the ring $E=\operatorname{End}_{A}\left(Y_{k}\right)$ has no non-zero nilpotent elements (i.e. it is reduced). Then the chain $Y=Y_{0} \supsetneq$ $Y_{1} \supsetneq \cdots \supsetneq Y_{k}$ satisfies condition iii) by construction, and condition i) by Lemma 17.c). By $[13,54.1]$ the reduced ring $E$ is completely reducible, hence $E$ is a finite direct product of division rings. It follows that the decomposition of $Y_{k}$ into the direct sum of indecomposable modules consists of bricks, and there are no non-zero homomorphisms between different members of the decomposition, so ii) holds.

Using Theorem 7, we obtain a result which generalizes [8, Proposition 5.6].

Corollary 19. Let $A$ be a right hereditary ring and $Y$ be a non-zero module of finite length. Then there exists a chain of submodules $Y=Y_{0} \supsetneq Y_{1} \supsetneq \cdots \supsetneq Y_{k}$ such that

i) $Y^{\perp}=Y_{1}^{\perp}=\cdots=Y_{k}^{\perp}$ 
ii) $Y_{k}=\bigoplus_{i=1}^{m} Z_{i}$ where each $Z_{i}$ is a brick such that $\operatorname{Hom}\left(Z_{i}, Z_{j}\right)=0$ for all $i \neq j$,

iii) $Y_{j} / Y_{j+1}$ embeds into $Y_{j}$ for all $j \in\{0, \ldots, k-1\}$.

For the proof of the main results we recall that the endomorphism ring of each indecomposable module of finite length is local [13, 32.4(3)], so by the Krull-Schmidt Theorem, every finite length module has a unique indecomposable decomposition (where uniqueness is understood up to order and isomorphism of the indecomposable factors).

Recall that a module $X$ is $S$-filtered if $X$ possesses a strictly increasing continuous chain $\left(S_{\alpha} \mid \alpha \leq \sigma\right)$ of submodules of $X$ such that $S_{\alpha+1} / S_{\alpha} \cong S$ for all $\alpha<\sigma$.

By [4], for each module $S$ there exists an $S$-filtered module $X$ with $\operatorname{Ext}_{A}^{1}(S, X)=$ 0 . The module $X$ can be used to test for vanishing of $\operatorname{Ext}_{A}^{1}(S, S)$ in the case when $S$ is simple:

Lemma 20. Let $X$ be any module, and $S$ be any simple submodule of $X$ such that $\operatorname{Ext}_{A}^{1}(S, X)=0$.

Then $\operatorname{Ext}_{A}^{1}(S, S)=0$ if and only if $N \cong S^{2}$ for each $S$-filtered submodule $N$ of $X$ with $\lg (N)=2$.

Proof. Assume that $N$ is an $S$-filtered submodule of $X$ such that $\lg (N)=2$ and $N \nsubseteq S^{2}$. Then the exact sequence $0 \rightarrow \operatorname{Soc}(N) \rightarrow N \rightarrow S \rightarrow 0$ is non-split, so $\operatorname{Ext}_{A}^{1}(S, S) \neq 0$.

Conversely, assume $\operatorname{Ext}_{A}^{1}(S, S) \neq 0$, and consider a non-split exact sequence $0 \rightarrow S \stackrel{\mu}{\rightarrow} T \rightarrow S \rightarrow 0$. By assumption, there is an embedding $\nu: S \rightarrow X$. We form the pushout of $\mu$ and $\nu$ :

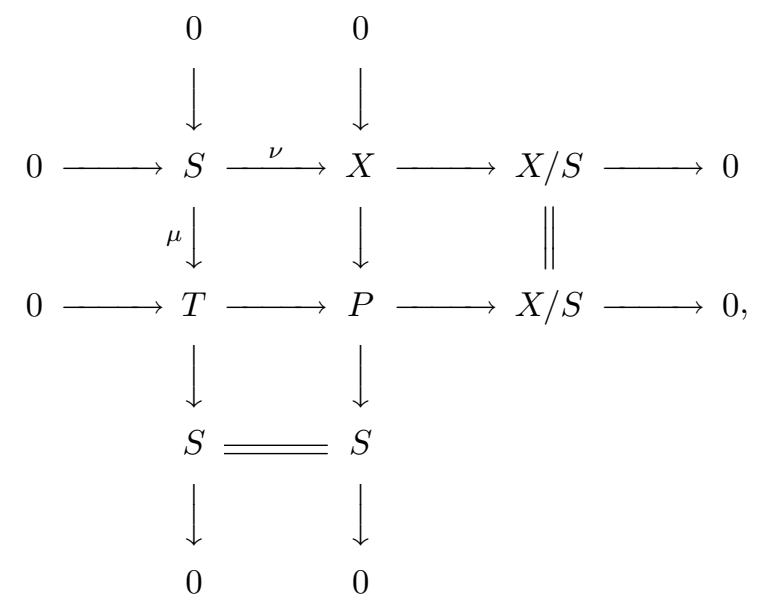

W.l.o.g., $T$ is a submodule of $P$. Since $\operatorname{Ext}_{A}^{1}(S, X)=0$, the second column splits, hence $P=X \oplus Y$ with $Y \cong S$. Let $\pi: P \rightarrow X$ be the projection. By the assumption on $N$, the restriction $\pi \uparrow T: T \rightarrow X$ is not a monomorphism. Since $\operatorname{Ker}(\pi)=Y$ is simple, we have $Y \subseteq T$, so $Y$ is a direct summand in $T$. This implies that $T \cong S^{2}$, a contradiction.

Theorem 21. Let $A$ be a ring. The following are equivalent for a non-zero module $X$ of finite length:

a) $X \cong Y$ whenever $Y$ has finite length, ${ }^{\circ} X={ }^{\circ} Y$, and $\lg (X)=\lg (Y)$; 
b) (I) $X \cong \bigoplus_{i=1}^{m} X_{i}$ where $X_{i}$ are bricks such that $\operatorname{Hom}\left(X_{i}, X_{j}\right)=0$ for all $i \neq j$, or

(II) $X \cong S^{r} \oplus \bigoplus_{i=1}^{m} X_{i}$ and

i) $r>0$ and $S$ is a simple module,

ii) $X_{1}, \ldots, X_{m}$ are bricks such that $\operatorname{Hom}\left(X_{i}, X_{j}\right)=0$ for all $i \neq j$,

iii) If $W$ is a submodule of $\bigoplus_{i=1}^{m} X_{i}$ such that $s=\lg (W) \leq r$ then $W \cong S^{s}$

iv) $\operatorname{Ext}_{A}^{1}\left(S, X_{i}\right)=0$ for each $i$.

Proof. a) $\Rightarrow$ b) By Lemma 18, there exists a submodule $X^{\prime}=\bigoplus_{i=1}^{m} X_{i}$ of $X$ such that ${ }^{\circ} X={ }^{\circ} X^{\prime}$, all $X_{i}$ s are bricks and $\operatorname{Hom}\left(X_{i}, X_{j}\right)=0$ whenever $i \neq j$. If $\lg (X)=\lg \left(X^{\prime}\right)$ then $X=X^{\prime}$ and we are in case (I).

Assume $r=\lg (X)-\lg \left(X^{\prime}\right)>0$. If $S$ is any simple submodule of $X^{\prime}$ then ${ }^{\circ} X={ }^{\circ} Y$ where $Y=S^{r} \oplus X^{\prime}$. So $X \cong S^{r} \oplus \bigoplus_{i=1}^{m} X_{i}$ by a). Thus we are in case (II) and the conditions i) and ii) hold.

Moreover, it follows that $S$ is the only simple module, up to isomorphism, that embeds into $X^{\prime}$, and the socle of every $X_{i}$ is a finite direct sum of copies of $S$.

Let $W$ be a submodule of $\bigoplus_{i=1}^{m} X_{i}$ such that $s=\lg (W) \leq r$. Then ${ }^{\circ} Y=$ ${ }^{\circ}\left(\bigoplus_{i=1}^{m} X_{i}\right)={ }^{\circ} X$ for $Y=W \oplus S^{r-s} \oplus \bigoplus_{i=1}^{m} X_{i}$, so $W \cong S^{s}$ by a). This proves condition iii).

If $\operatorname{Ext}^{1}\left(S, X_{j}\right) \neq 0$, then there is a non-split short exact sequence $0 \rightarrow X_{j} \rightarrow$ $M \stackrel{\beta}{\rightarrow} S \rightarrow 0$. Since $S$ is simple, $M \nsubseteq S \oplus X_{j}$. Note that $X_{j}$, and hence $M$, has a submodule isomorphic to $S$. So we can view $\beta$ as an endomorphism of $M$ such that $\beta^{2}=0$. Then ${ }^{\circ} M={ }^{\circ}\left(X_{j} \oplus S\right)$ by Lemma 17.c $)$. So ${ }^{\circ} X={ }^{\circ}\left(S^{r-1} \oplus M \oplus \bigoplus_{i \neq j} X_{i}\right)$, and $M \cong S \oplus X_{j}$ by a), a contradiction. This proves that $\operatorname{Ext}^{1}\left(S, X_{j}\right)=0$ for all $j$.

b) $\Rightarrow$ a) The case (I) has been proved in Theorem 13 . We will prove the case (II).

Let $X$ be as in b)(II), and $Y$ be a module such that ${ }^{\circ} X={ }^{\circ} Y$ and $\lg (X)=\lg (Y)$. Note that the socle of $X$ is isomorphic to a direct sum of copies of $S$, by iii). Then $Y$ has the same property: otherwise there exists a simple module $T ¥ S$ and a non-zero homomorphism $T \rightarrow Y$, but $\operatorname{Hom}(T, X)=0$.

Moreover, we claim that each submodule of $Y$ of length $\leq r$ is isomorphic to a direct sum of copies of $S$. We have just proved this for $r=1$. So suppose that $U$ is a submodule of $Y$ such that $\lg (U) \leq r$ and $r \geq 2$. Then there exists a non-zero homomorphism $f_{1}: U \rightarrow X$. Since $\lg \left(\operatorname{Im}\left(f_{1}\right)\right) \leq r$, condition iii) gives that $\operatorname{Im}\left(f_{1}\right)$ is isomorphic to a finite direct sum of copies of $S$. Suppose $U_{1}=\operatorname{Ker}\left(f_{1}\right) \neq 0$. Hence $\operatorname{Hom}\left(\operatorname{Ker}\left(f_{1}\right), Y\right) \neq 0$, and there exists a non-zero homomorphism $f_{2}: U_{1} \rightarrow X$. Repeating the previous arguments we find a chain of submodules $U=U_{0} \supsetneq U_{1} \supsetneq \cdots \supsetneq U_{k} \supsetneq \cdots$ and a family of homomorphisms $f_{i}: U_{i-1} \rightarrow X$ such that $U_{i}=\operatorname{Ker}\left(f_{i}\right)$. Since $U$ has finite length, there exists $k$ such that $U_{k}=0$, i.e. $f_{k}$ is a monomorphism. Then $U_{k-1}$ is a finite direct sum of copies of $S$. Then the exact sequence $0 \rightarrow U_{k-1} \rightarrow U_{k-2} \stackrel{f_{k-1}}{\rightarrow} \operatorname{Im}\left(f_{k-1}\right) \rightarrow 0$ splits since $\operatorname{Ext}\left(\operatorname{Im}\left(f_{k-1}\right), U_{k-1}\right)=0$ by Lemma 20 . It follows that $U_{k-2}$ is a direct sum of copies of $S$. Repeating this argument we obtain that all $U_{i}$ s are finite direct sums of copies of $S$, and the claim is proved.

Finally, consider a sequence of submodules $Y=Y_{0}>Y_{1}>\cdots>Y_{k}$ as in Lemma 18. Then ${ }^{\circ}\left(\bigoplus_{i=1}^{m} X_{i}\right)={ }^{\circ} Y_{k}$ and using condition ii) and Theorem 13, we obtain that $\bigoplus_{i=1}^{m} X_{i} \cong Y_{k}$. Notice that for each $i \in\{0, \ldots, k-1\}$, the module $Y_{i} / Y_{i+1}$ is of length $\leq r$ and it embeds into $Y$ by Lemma 18. By the claim above, 
we infer that every module $Y_{i} / Y_{i+1}$ is isomorphic to a finite direct sum of copies of $S$. If $r=1$ then $k=1$ and $Y / Y_{1} \cong S$, hence $Y \cong Y_{k} \oplus S$ by condition iv). If $r>1$ then $Y / Y_{k}$ is a direct sum of copies of $S$ by Lemma 20 , so again $Y \cong S^{r} \oplus Y_{k} \cong X$ by condition iv).

If $A$ is any ring and $\mathcal{S}$ any non-empty finite set of non-isomorphic simple modules, then the module $X=\bigoplus_{S \in \mathcal{S}} S$ satisfies condition b)(I) of Theorem 21.

Moreover, if $S$ is a simple module and $n>1$ then $X=S^{n}$ satisfies condition a) if and only if $\operatorname{Ext}_{A}^{1}(S, S)=0$, by condition b)(II) (here we consider $r=n-1$ ).

Given $r>0$ and $m>0$, we will now present an example of a ring $A$ and a module $X$ satisfying condition b)(II) for these $r$ and $m$ :

Example 22. Let $K$ be a field and let $\left(r_{i} \mid i=1, \ldots, m\right)$ be a sequence of integers such that $r_{i} \geq r$ for all $i$. Let $A$ be the finite dimensional hereditary $K$-algebra consisting of all $(m+1) \times(m+1)$ upper triangular matrices of the form

$$
A=\left(\begin{array}{ccc}
K & \Theta & K^{r_{1}} \\
\dddot{\Theta} & \dddot{K} & \dddot{M}_{r_{m}} \\
\Theta & 0 & K
\end{array}\right)
$$

where $\Theta$ denotes the $1 \times(m-1)$ matrix all of whose entries are zero. In other words, if we denote by $Q_{i}$ the quiver of the generalized $r_{i}$-Kronecker algebra (such $Q_{i}$ has only two vertices: a source and a sink, and $r_{i}$ arrows), then $A$ is the path algebra of the quiver obtained by identifying the sinks of the quivers $Q_{i}$ for all $i=1, \ldots, m$.

For $i=1, \ldots, m+1$ let $e_{i}$ be the $i$-th diagonal matrix unit (so $e_{i}$ has exactly one non-zero entry, namely 1 in $i$-th row and $i$-th column). Then $\left\{e_{i} \mid i=1, \ldots, m+1\right\}$ is a complete set of primitive idempotents of $A$. Moreover, $S=e_{m+1} A$ is the unique projective simple module.

For $i=1, \ldots, m$, the projective module $X_{i}=e_{i} A$ has length $r_{i}+1$, and its socle is isomorphic to $S^{r_{i}}$. If $W$ is a submodule of $\bigoplus_{i=1}^{m} X_{i}$ of length $s \leq r$, then $W$ is projective, hence a direct sum of some copies of $S$ and $X_{i}(i=1, \ldots, m)$. But each $X_{i}$ has length $>r$, so no $X_{i}$ occurs in the decomposition of $W$, and $W \cong S^{s}$. Since $\operatorname{End}_{A}\left(X_{i}\right) \cong e_{i} A e_{i} \cong K$, it is easy to see that $X=S^{r} \oplus \bigoplus_{i=1}^{m} X_{i}$ satisfies conditions i)-v) of Theorem 21.b)(II) for the given $r$ and $m$.

In the following example we will show that the conditions ii)-iv) in Theorem 21.b)(II) are independent.

Example 23. (a) Let $A$ be a commutative local QF-ring which is not a field. If $S$ the simple $A$-module, then $X=S \oplus A$ satisfies iii) and iv), but not ii).

(b) An example where only iii) fails comes from a modification of Example 22: iii) will fail for the $X$ defined there if $r_{i}<r$ for some $i$. Alternatively, we could take any non-completely reducible ring $A$ which has a simple projective module $S$, and let $\left\{X_{1}, \ldots, X_{m}\right\}$ be a finite set of non-projective pairwise non-isomorphic simple modules.

(c) To show that iv) is independent it is enough to consider a ring $A$ possessing a simple module $S$ such that $\operatorname{Ext}_{A}^{1}(S, S) \neq 0$, and take $X=S^{r} \oplus S$.

Returning to the annihilator classes of the form $X^{\perp}$, we first observe that if the ring $A$ is as in Theorem 8 then Theorem 21 also characterizes the finite length modules $X$ with the property that $X \cong Y$ for each finite length module $Y$ such that $X^{\perp}=Y^{\perp}$ and $\lg (X)=\lg (Y)$. For the remaining case, the solution is given by the following theorem: 
Theorem 24. Let $A$ be a right hereditary ring with at least two non-isomorphic simple modules, and $X$ be a module of finite length.

(I) If A has at least two non-isomorphic projective simple modules then the following are equivalent:

a) $X \cong Y$ whenever $Y$ has finite length, $X^{\perp}=Y^{\perp}$, and $\lg (X)=\lg (Y)$;

b) $X \cong \bigoplus_{i=1}^{m} X_{i}$ is a direct sum of finite length non-projective bricks such that $\operatorname{Hom}\left(X_{i}, X_{j}\right)=0$ for all $i \neq j$.

(II) If up to isomorphism $A$ has exactly one projective simple module $S$, then the following are equivalent:

a) $X \cong Y$ whenever $Y$ has finite length, $X^{\perp}=Y^{\perp}$, and $\lg (X)=\lg (Y)$;

b) $X \cong S^{r} \oplus \bigoplus_{i=1}^{m} X_{i}$ for some $r \geq 0$, and

i) all projective modules of length $s \leq r$ are isomorphic to $S^{s}$,

ii) each $X_{i}$ is a finite length non-projective brick and $\operatorname{Hom}\left(X_{i}, X_{j}\right)=$ 0 for all $i \neq j$,

iii) $U \cong S^{s}$ whenever $U$ is a submodule of $\bigoplus_{i=1}^{m} X_{i}$ of length $s \leq r$.

Proof. a) $\Rightarrow$ b) Let $X$ be a finite length module as in conditions (I) a) or (II) a). Without loss of generality, we can suppose that $X$ is not projective. By Corollary 19 , there exists a submodule $X^{\prime} \leq X$ such that $X^{\prime}=\bigoplus_{i=1}^{m} X_{i}$ is a direct sum of finite length non-projective bricks such that $\operatorname{Hom}\left(X_{i}, X_{j}\right)=0$ if $i \neq j$, and $X^{\prime \perp}=X^{\perp}$. Let $r=\lg (X)-\lg \left(X^{\prime}\right)$.

If $S$ and $T$ are simple projective modules then $X^{\perp}=\left(S^{r} \oplus X^{\prime}\right)^{\perp}=\left(T^{r} \oplus X^{\prime}\right)^{\perp}$, hence $X \cong S^{r} \oplus X^{\prime} \cong T^{r} \oplus X^{\prime}$ by a). So in the case (I), $X=X^{\prime}$, and b) holds.

In the case (II), we obtain $X=S^{r} \oplus \bigoplus_{i=1}^{m} X_{i}$ where all $X_{i}$ s are finite length non-projective bricks with $\operatorname{Hom}\left(X_{i}, X_{j}\right)=0$ for all $i \neq j$. Let $U$ be a submodule of $\bigoplus_{i=1}^{m} X_{i}$ of length $s \leq r$. Since $A$ is hereditary, $\left(\bigoplus_{i=1}^{m} X_{i}\right)^{\perp} \subseteq U^{\perp}$, hence $X^{\perp}=\left(S^{r-s} \oplus U \oplus \bigoplus_{i=1}^{m} X_{i}\right)^{\perp}$, and $U \cong S^{s}$. Similarly, each projective module of finite length $q \leq r$ is isomorphic to $S^{q}$. This proves b).

b) $\Rightarrow$ a) In the case (I), the implication is a consequence of Theorem 15 .

In the case (II), let $Y$ be a module such that $X^{\perp}=Y^{\perp}$. Again by Corollary 19, there exists a submodule $Y^{\prime} \leq Y$ such that $Y^{\prime}=\bigoplus_{i=1}^{n} Y_{i}$ is a direct sum of finite length non-projective bricks, $\operatorname{Hom}\left(Y_{i}, Y_{j}\right)=0$ for $i \neq j$, and $Y^{\perp}=Y^{\perp}=X^{\perp}=$ $X^{\prime \perp}$, where $X^{\prime}=\bigoplus_{i=1}^{m} X_{i}$. By Theorem 15 , we have $m=n$ and without loss of generality we can assume $Y_{i} \cong X_{i}$ for all $i \in\{1, \ldots, m\}$. By Lemma 5 there exists a descending chain $Y=Z_{0} \supsetneq Z_{1} \supsetneq \cdots \supsetneq Z_{k}$ such that $Z_{k}$ is projective, and for each $i \in\{0, \ldots, k-1\}$ there exist an index $j_{i} \in\{1, \ldots, m\}$ and a homomorphism $\varphi_{i}: Z_{i} \rightarrow X_{j_{i}}$ such that $\operatorname{Ker}\left(\varphi_{i}\right)=Z_{i+1}$.

Let $j \in\{1, \ldots, m\}$. Since $Y_{j}$ is not projective, there exists $i \in\{0, \ldots, k-1\}$ such that $Y_{j} \subseteq Z_{i}$, but $Y_{j} \nsubseteq Z_{i+1}$. Then the restriction $\varphi_{i}\left\lceil Y_{j}: Y_{j} \rightarrow X_{j_{i}}\right.$ is a non-zero homomorphism, hence $j_{i}=j$ and $\varphi_{i}\left\lceil Y_{j}\right.$ is an isomorphism. Then $\varphi_{i}$ is a split epimorphism, and $Z_{i}=Z_{i+1} \oplus U_{i}$ where $U_{i} \cong Y_{j} \cong X_{j}$.

So for every $j \in\{1, \ldots, m\}$ we can fix an index $i_{j} \in\{0, \ldots, k-1\}$ such that $Z_{i_{j}}=$ $Z_{i_{j}+1} \oplus U_{j}$ with $U_{j} \cong X_{j}$. Since $X_{j} ¥ X_{j^{\prime}}$ whenever $j \neq j^{\prime}$, the correspondence $j \mapsto i_{j}$ is $1-1$.

Let $i \in\{0, \ldots, k-1\} \backslash\left\{i_{1}, \ldots, i_{m}\right\}$. Since $\sum_{i=0}^{k-1} \lg \left(Z_{i} / Z_{i+1}\right)+\lg \left(Z_{k}\right)=\lg (Y)=$ $\lg (X)=r+\sum_{j=1}^{m} \lg \left(X_{j}\right)$, we have $r_{i}=\lg \left(Z_{i} / Z_{i+1}\right) \leq r$, hence $\operatorname{Im}\left(\varphi_{i}\right) \cong S^{r_{i}}$ is projective. Then $Z_{i}=Z_{i+1} \oplus U_{i}$, where $U_{i} \cong S^{r_{i}}$. 
Moreover, we observe that iii) implies that $\lg \left(X_{j}\right)>r$ for all $j$. So the index $i_{j}$ is uniquely determined by $j$ since otherwise we would obtain, using the formula $\sum_{i=0}^{k-1} \lg \left(Z_{i} / Z_{i+1}\right)+\lg \left(Z_{k}\right)=\lg (Y)$, that $\lg (Y)>\lg (X)$.

It follows that for all $i \in\{0, \ldots, k-1\}$ there exists a direct sum decomposition $Z_{i}=Z_{i+1} \oplus U_{i}$ such that for every $j \in\{1, \ldots, m\}$ we can find exactly one index $i_{j} \in\{0, \ldots, k-1\}$ with $U_{i_{j}} \cong X_{j}$, and $U_{i} \cong S^{r_{i}}$ for all $i \notin\left\{i_{1}, \ldots, i_{m}\right\}$. Then $Y=U_{0} \oplus \cdots \oplus U_{k-1} \oplus Z_{k} \cong X^{\prime} \oplus S^{t} \oplus Z_{k}$. Since $\lg (Y)=\lg (X), Z_{k}$ is a projective module of finite length equal to $r-t$, so i) gives that $Z_{k} \cong S^{r-t}$. This proves that $Y \cong X^{\prime} \oplus S^{r} \cong X$.

Let $A$ be any right hereditary ring with at least two non-isomorphic projective simple modules. If $\mathcal{S}$ is any finite non-empty set of non-isomorphic non-projective simple modules then the module $X=\bigoplus_{S \in \mathcal{S}} S$ clearly satisfies condition (I) b) of Theorem 24.

Finally, given $r>0$ and $m>0$, we will present an example of a hereditary ring $A$ and a module $X$ satisfying condition (II) b) for these $r$ and $m$ :

We consider the algebra $A$ from Example 22, but we require $r_{i}>r$ for all $i=1, \ldots, m$. Again, we take $S=e_{m+1} R$, the unique simple projective module, but for $i=1, \ldots, m$, we replace $X_{i}$ by $\overline{X_{i}}$, its factor modulo a simple submodule. Let $X=S^{r} \oplus \bigoplus_{i=1}^{m} \overline{X_{i}}$. Then condition i) holds because each projective module is a direct sum of some copies of $S$ and of the $X_{i} \mathrm{~s}(i=1, \ldots, m)$, and $\lg \left(X_{i}\right)>r$ for all $i$. Since $\overline{X_{i}}$ is generated by the coset of the idempotent $e_{i}$, we infer that $\operatorname{End}_{A}\left(\overline{X_{i}}\right)=K$ for all $i=1, \ldots, m$, and it is easy to see that condition ii) holds. Finally, each submodule of $\bar{X}=\bigoplus_{i=1}^{m} \overline{X_{i}}$ of length $s \leq r$ is contained in the socle of $\bar{X}$, so condition iii) also holds.

In the end we mention that the dual statements for Lemma 17, Lemma 18 and Theorem 21 are true. However, a dual of Theorem 24 is not available, as a consequence of Example 28.

Lemma 25. Let $A$ be a ring, and $X, Y$, and $Z$ be modules.

a) $(X \oplus Y)^{\circ}=X^{\circ} \cap Y^{\circ}$;

b) If $0 \rightarrow X \rightarrow Y \rightarrow Z \rightarrow 0$ is a short exact sequence then $(X \oplus Z)^{\circ} \subseteq Y^{\circ} \subseteq$ $Z^{\circ}$

c) If $f \in \operatorname{End}(X)$ and $H=\operatorname{Im}(f)$ then $X^{\circ}=(H \oplus X / H)^{\circ}$. If moreover $f^{2}=0$ then also $X^{\circ}=(X / H)^{\circ}$.

Lemma 26. Let $A$ be a ring and $Y$ be a non-zero module of finite length. Then there exists a chain of submodules $0=Y_{0} \subsetneq Y_{1} \subsetneq \cdots \subsetneq Y_{k}$ such that

i) $Y^{\circ}=\left(Y / Y_{1}\right)^{\circ}=\cdots=\left(Y / Y_{k}\right)^{\circ}$,

ii) $Y / Y_{k}=\bigoplus_{i=1}^{m} Z_{i}$ where each $Z_{i}$ is a brick such that $\operatorname{Hom}\left(Z_{i}, Z_{j}\right)=0$ for all $i \neq j$.

iii) Every $Y_{i+1} / Y_{i}$ is an epimorphic image of $Y$.

Proof. We proceed in the same way as in the proof of Lemma 18: Suppose that a submodule $Y_{i}$ has been constructed. If $Y / Y_{i}$ has no non-zero nilpotent endomorphisms we take $k=i$. Otherwise there exists an endomorphism $0 \neq f: Y / Y_{i} \rightarrow$ $Y / Y_{i}$ such that $f^{2}=0$, and we put $Y_{i+1} \leq Y$ the only submodule such that $Y_{i+1} / Y_{i}=\operatorname{Im}(f)$.

Theorem 27. Let $A$ be a ring. The following are equivalent for a non-zero module $X$ of finite length: 
a) $X \cong Y$ whenever $Y$ has finite length, $X^{\circ}=Y^{\circ}$, and $\lg (X)=\lg (Y)$;

b) (I) $X \cong \bigoplus_{i=1}^{m} X_{i}$ where $X_{i}$ are bricks such that $\operatorname{Hom}\left(X_{i}, X_{j}\right)=0$ for all $i \neq j$, or

(II) $X \cong S^{r} \oplus \bigoplus_{i=1}^{m} X_{i}$ and

i) $r>0$ and $S$ is a simple module,

ii) $X_{1}, \ldots, X_{m}$ are non-simple bricks such that $\operatorname{Hom}\left(X_{i}, X_{j}\right)=0$ for all $i \neq j$,

iii) If $W$ is an epimorphic image of $\bigoplus_{i=1}^{m} X_{i}$ such that $s=\lg (W) \leq$ $r$ then $W \cong S^{s}$

iv) $\operatorname{Ext}_{A}^{1}\left(X_{i}, S\right)=0$ for each $i$.

Proof. The proof is dual to the proof of Theorem 21. We present some details about the last part of it.

Consider a sequence of submodules $0=Y_{0}<Y_{1}<\cdots<Y_{k}$ as in Lemma 26 . Then $\left(\bigoplus_{i=1}^{m} X_{i}\right)^{\circ}=\left(Y / Y_{k}\right)^{\circ}$ and using condition ii) and Theorem 14, we obtain that $\bigoplus_{i=1}^{m} X_{i} \cong Y / Y_{k}$. Then $\lg \left(Y_{k}\right)=r$. Therefore, for each $i \in\{0, \ldots, k-1\}$, the module $Y_{i+1} / Y_{i}$ is of length $\leq r$ and it is an epimorphic image of $Y$ by Lemma 26. Then every module $Y_{i+1} / Y_{i}$ is isomorphic to a finite direct sum of copies of $S$.

If $r=1$ then $k=1$ and $Y_{1} \cong S$, hence $Y \cong\left(Y / Y_{1}\right) \oplus S$ by condition iv).

If $r>1$ then $Y_{1} \cong Y_{1} / Y_{0}$ is isomorphic to a direct sum of copies of $S$. Suppose that $Y_{i}$ is isomorphic to a direct sum of copies of $S$. Since the exact sequence $0 \rightarrow Y_{i} \rightarrow Y_{i+1} \rightarrow Y_{i+1} / Y_{i} \rightarrow 0$ splits by iv), $Y_{i+1}$ is isomorphic to a direct sum of copies of $S$. Then $Y_{k} \cong S^{r}$, hence $Y \cong S^{r} \oplus Y / Y_{k} \cong X$ by condition iv).

If $A$ and $B$ are rings, we will denote by $A \boxplus B$ the direct product (in the category of all rings) of $A$ and $B$.

Example 28. Let $A$ be the ring from Example 12 and $k$ be a field.

The ring $A \boxplus k \boxplus k$ has two simple injective modules, but by Remark 16, under $\mathrm{V}=\mathrm{L}$, it has two non-injective simple modules $S_{1}$ and $S_{2}$ such that ${ }^{\perp} S_{1}={ }^{\perp} S_{2}$. So the dual of Theorem 24(I) is not provable for $X=S_{1}$.

Similarly, we take the ring $A \boxplus k$ to see that the dual of Theorem 24(II) in not provable for $r=0$ and $X=S_{1}$.

\section{REFERENCES}

[1] Assem, I., Simson, D., Skowronski, A., Elements of the Representation Theory of Associative Algebras I, Cambridge Univ. Press, Cambridge 2006.

[2] Colpi, R., Tonolo, A., Trlifaj, J., Perpendicular categories of infinite dimensional partial tilting modules and transfers of tilting torsion classes, J. Pure Appl. Algebra 211(2007), 223-234.

[3] Eklof, P.C., Mekler, A.H., Almost Free Modules, Revised ed., North-Holland, New York 2002.

[4] Eklof, P.C., Trlifaj, J., How to make Ext vanish, Bull. London Math. Soc. 33(2001), 41-51.

[5] Göbel, R., Trlifaj, J., Cotilting and a hierarchy of almost cotorsion groups, J. Algebra 224(2000), 110-122.

[6] Göbel, R., Trlifaj, J., Approximations and Endomorphism Algebras of Modules, W. de Gruyter, Berlin 2006.

[7] Goodearl, K., Von Neumann Regular Rings, Krieger, Malabar (FL) 1991.

[8] Kerner, O., Trlifaj, J., Tilting classes over wild hereditary algebras, J. Algebra 290(2005), 538-556.

[9] Stenström, B., Rings of Quotients, Springer-Verlag, New York 1975.

[10] Trlifaj, J., Similarities and differences between abelian groups and modules over non-perfect rings, Contemp. Math. 171(1994), 397-406.

[11] Trlifaj, J., Whitehead test modules, Trans. Amer. Math. Soc. 348(1996), 1521-1554. 
[12] Wickless, W. J., An equivalence relation for torsion-free abelian groups of finite rank, J. Algebra 153(1992), 1-12.

[13] Wisbauer, R., Foundations of Module and Ring Theory, Gordon \& Breach, Philadelphia 1991.

"Babes-Bolyai" University, Faculty of Mathematics and Computer Science, Str. Mihail KogăLniceanu 1, 400084 Cluj-Napoca, Romania

E-mail address: bodo@math.ubbcluj.ro

Department of Algebra, Faculty of Mathematics and Physics, Charles University, Sokolovská 83, 18675 Prague, Czech Republic

E-mail address: trlifaj@karlin.mff.cuni.cz 